\title{
Transition-Metal-Free Synthesis of Poly(phenylene ethynylene)s with Alternating Aryl- Perfluoroaryl Units
}

Tanmoy Dutta, Kathy B. Woody, Mark D. Watson

Dept. of Chemistry, University of Kentucky, Lexington, KY USA 40506-0055

mdwatson@uky.edu

\section{Supporting Information:}

Materials and Methods: CsF, 18-Crown-6, and Tetramethylammonium fluoride (TMAF) were dried under reduced pressure $\left(<10^{-3} \mathrm{mbar}\right)$ at $180^{\circ} \mathrm{C}, 80^{\circ} \mathrm{C}$ and $130^{\circ} \mathrm{C}$ respectively and stored in an argon-filled glove box. $\mathrm{C}_{6} \mathrm{~F}_{6}$, toluene, ether, DMF, and THF were distilled from appropriate drying agents and stored over molecular sieves under argon. "Anhydrous" Tetra-n-butylammonium fluoride (TBAF) (1M solution in THF, $\sim 5 \%$ water) and zinc chloride anhydrous beads were purchased from Aldich and stored in an argon-filled glove box. All other materials were used as purchased. Unless otherwise stated, all manipulations and reactions were carried out under argon in a glove box or using standard Schlenk techniques. ${ }^{1} \mathrm{H},{ }^{13} \mathrm{C},{ }^{19} \mathrm{~F}$ NMR spectra were collected on a Varian INOVA $400 \mathrm{MHz}$ or $200 \mathrm{MHz}$ spectrometer (purchased under the CRIF Program of the NSF grant CHE-9974810). Chemical shifts were referenced to residual protio-solvent signals, except for ${ }^{19} \mathrm{~F}$ NMR spectra, where $\mathrm{CCl}_{3} \mathrm{~F}$ was added as internal standard and set to $\delta=0$ ppm. Relative molecular weight determinations were performed using a Waters 600E HPLC system, driven by Waters Empower Software and equipped with two linear mixed-bed GPC columns (American Polymer Standards Corporation, AM Gel Linear/15) in series. Eluting polymers were detected with both refractive index and photodiode array detectors. The system was calibrated with 11 narrow PDI polystyrene samples in the range $580-2 \mathrm{X} 10^{6} \mathrm{Da}$ with THF at a flow rate $1 \mathrm{ml} / \mathrm{min}$. Differential Scanning Calorimetry was performed using a nitrogen-purged Mettler DSC $822^{\mathrm{e}}$ with scanning rates of $10^{\circ} \mathrm{C} / \mathrm{min}$. GC-MS data were collected using an Agilent technologies $6890 \mathrm{~N}$ GC with 5973 MSD. High resolution mass spectra were provided by the University of Kentucky Mass Spectrometry facility.

Tetra-alkoxy benzenes and monomer 1a were prepared via published procedures. ${ }^{[1,2]}$

Synthesis and Characterization

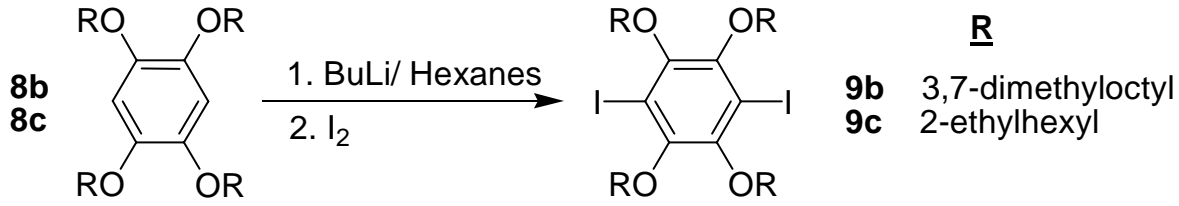

General Procedure for iodination of tetra-alkoxy benzene:In an oven-dried Schlenk flask, BuLi ( 3 eq, $1.6 \mathrm{M}$ hexanes) were added dropwise to a solution of $\mathbf{8}$ in dry hexanes under argon atmosphere. After 48 hours, excess iodine was added to the solution and the whole was stirred for 12 hours. The reaction mixture was diluted with pentane and washed with $10 \% \mathrm{KOH}(\mathrm{aq})$ followed by DI-water to remove unreacted iodine. The combined organic extracts were dried over $\mathrm{MgSO}_{4}$, filtered, concentrated under reduced pressure, and purified via column chromatography.

1,2,4,5-tetrakis(3,7-dimethyloctyloxy)-3,6-diiodobenzene (9b): Compound 8b (8g, $11.4 \mathrm{mmol}) \mathrm{BuLi}(21.33 \mathrm{ml}, 1.6 \mathrm{M}$ hexanes 34.1 mol), iodine (14.43g, $56.8 \mathrm{mmol})$. Colorless liquid $(9.38 \mathrm{~g}, 86.4 \%) .{ }^{1} \mathrm{H}$ NMR $\left(400 \mathrm{MHz}, \mathrm{CDCl}_{3}\right) \delta: 3.97(\mathrm{~m}, 8 \mathrm{H}), 1.86(\mathrm{~m}, 4 \mathrm{H}), 1.63(\mathrm{~m}, 12 \mathrm{H}), 1.27$ $(\mathrm{m}, 24 \mathrm{H}), 0.85(\mathrm{~m}, 36 \mathrm{H}){ }^{13} \mathrm{C}$ NMR $\left(100 \mathrm{MHz}, \mathrm{CDCl}_{3}\right) \delta: 148.96,92.78,72.27,39.28,37.35,37.34,37.28,29.8,27.96,24.67,24.7,22.72$, $22.61,19.75$.

1,2,4,5-tetrakis(2-ethylhexyloxy)-3,6-diiodobenzene (9c):

1,2,4,5-tetrakis(2-ethylhexyloxy)-benzene (8c) (5.00g, $8.46 \mathrm{mmol})$, BuLi (15.86ml, $1.6 \mathrm{M}$ hexanes $25.38 \mathrm{mmol})$, iodine (10.74g, 42.3 mmol). Colorless liquid (5.89g, 82.9\%). ${ }^{1} \mathrm{H}$ NMR $\left(200 \mathrm{MHz}, \mathrm{CDCl}_{3}\right) \delta: 3.78(\mathrm{~d}, 8 \mathrm{H}), 1.7(\mathrm{~m}, 4 \mathrm{H}), 1.43(\mathrm{~m}, 32 \mathrm{H}), 0.91(\mathrm{~m}, 24 \mathrm{H}){ }^{13} \mathrm{C} \mathrm{NMR}$ $\left(50 \mathrm{MHz}, \mathrm{CDCl}_{3}\right) \delta: 149.07,92.63,40.43,30.34,29.19,23.71,23.18,14.18,11.21$.

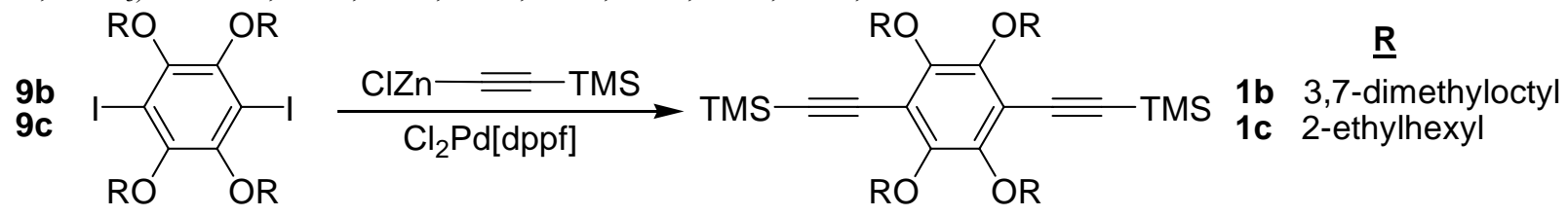

General Procedure for synthesis of monomers 1:

A solution of $\mathrm{n}$-BuLi was added slowly via syringe to an ice-cooled solution of trimethylsilylacetylene in dry THF. After stirring for 3 hours, anhydrous $\mathrm{ZnCl}_{2}$ was added to the flask. After stirring an additional 45 minutes, compound 9 was added and the solution was deoxygenated by sparging with argon, followed by the addition of $\mathrm{Cl}_{2} \mathrm{Pd}[\mathrm{dppf}] \cdot \mathrm{MeCl}_{2}$. The vessel was sealed and submersed in a $110^{\circ} \mathrm{C}$ oil bath for 48 hours. The contents were poured into water, extracted with pentane, and the combined organic extracts were dried over $\mathrm{MgSO}_{4}$, filtered, and concentrated under reduced pressure. Monomers 1 were then isolated via column chromatography (silica gel, pentane $/ \mathrm{CH}_{2} \mathrm{Cl}_{2}$ ).

1,2,4,5-tetrakis(3,7-dimethyloctyloxy)-3,6-bis(2-(trimethylsilyl)ethynyl)benzene(1b): BuLi (10.47 $\mathrm{mL}, 1.6 \mathrm{M}$ hexanes, $16.7 \mathrm{mmol}$ ), TMS-acetylene $(2.46 \mathrm{~mL}, 17.6 \mathrm{mmol}), \mathrm{ZnCl}_{2}(2.52 \mathrm{~g}, 18.8 \mathrm{mmol})$, compound $\mathbf{8 b}(4 \mathrm{~g}, 4.19 \mathrm{mmol}), \mathrm{Cl}_{2} \mathrm{Pd}[\mathrm{dppf}] \cdot \mathrm{MeCl}_{2}(171 \mathrm{mg}, 0.21$ mmol). Isolated by column chromatography (silica gel, pentane $\left./ \mathrm{CH}_{2} \mathrm{Cl}_{2} 5: 1\right)$ as a pale yellow oil $(2.46 \mathrm{~g}, 66 \%)$. ${ }^{1} \mathrm{H} \mathrm{NMR}\left(400 \mathrm{MHz}, \mathrm{CDCl}{ }_{3}\right)$ $\delta 4.027(\mathrm{~m}, 8 \mathrm{H}), 1.828(\mathrm{~m}, 4 \mathrm{H}), 1.64(\mathrm{~m}, 12 \mathrm{H}), 1.21(\mathrm{~m}, 24 \mathrm{H}), 0.904(\mathrm{~m}, 36 \mathrm{H}) \quad 0.226(\mathrm{~s}, 18 \mathrm{H}) .{ }^{13} \mathrm{C} \mathrm{NMR}(100 \mathrm{MHz} \mathrm{CDCl})_{3} \delta: 150.14$ 114.84, 104.24, 96.93, 72.63, 39.31, 37.53, 37.37, 29.75, 27.96, 24.68, 22.69, 22.59, 19.68, -0.063. Anal.Calcd for $\mathrm{C}_{56} \mathrm{H}_{102} \mathrm{O}_{4} \mathrm{Si}_{2}: \mathrm{C}_{\text {, } 75.1}$; H, 11.48. Found: C, 75.09; H, 11.11 .

1,2,4,5-tetrakis(2-ethylhexyloxy)-3,6-bis(2-(trimethylsilyl)ethynyl)benzene(1c): $\mathrm{BuLi}(14.2 \mathrm{~mL}, 1.6 \mathrm{M}$ hexanes, $22.7 \mathrm{mmol})$, TMSacetylene $(3.64 \mathrm{~mL}, 23.9 \mathrm{mmol}), \mathrm{ZnCl}_{2}(3.43 \mathrm{~g}, 25.6 \mathrm{mmol})$, compound $\mathbf{8 c}(4.8 \mathrm{~g}, 5.69 \mathrm{mmol}), \mathrm{Cl}_{2} \mathrm{Pd}[\mathrm{dppf}] \cdot \mathrm{MeCl}_{2}(233 \mathrm{mg}, 0.28 \mathrm{mmol})$. Isolated by column chromatography (silica gel, pentane $\left./ \mathrm{CH}_{2} \mathrm{Cl}_{2} 5: 1\right)$ as a pale yellow oil (2.62 g, 59\%). ${ }^{1} \mathrm{H} \mathrm{NMR} \mathrm{(400MHz,} \mathrm{CDCl} 3$ ) 
$\delta 3.88(\mathrm{~m}, 8 \mathrm{H}), 1.62(\mathrm{~m}, 4 \mathrm{H}), 1.48(\mathrm{~m}, 32 \mathrm{H}), 0.93(\mathrm{~m}, 24 \mathrm{H}) ,0.21(\mathrm{~s}, 18 \mathrm{H}) .{ }^{13} \mathrm{C} \mathrm{NMR}\left(100 \mathrm{MHz}, \mathrm{CDCl}_{3}\right) \delta 149.98,114.22,103.98$, 96.91, 76.09, 40.23, 29.99, 29.03, 23.42, 22.84, 13.84, 10.87,-0.36. Anal.Calcd for $\mathrm{C}_{48} \mathrm{H}_{86} \mathrm{O}_{2} \mathrm{Si}_{2}$ : C, 73.59; H, 11.07. Found: C, 74.2; H, 11.83.

General methods for polymer synthesis:

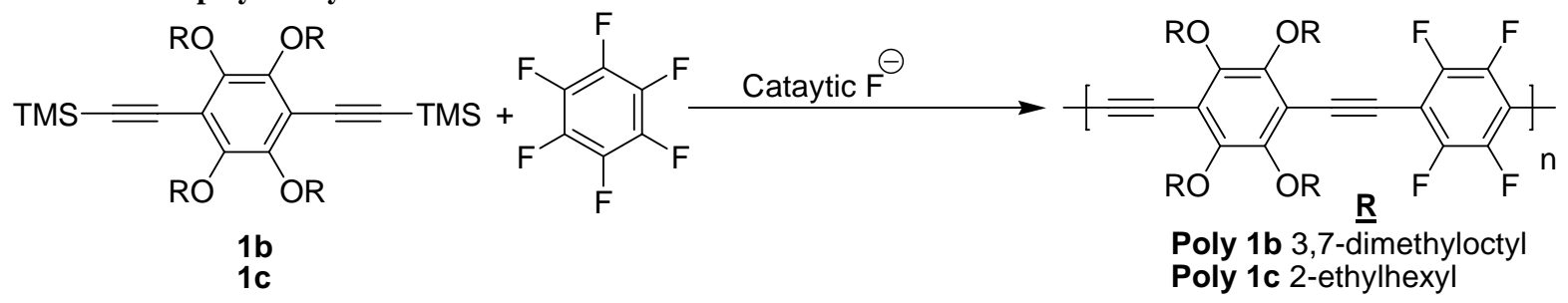

Method 1 (TBAF, anhydrous conditions).

In an argon-filled glove box, monomer $\mathbf{1 b}$ or $\mathbf{1 c}$, anhydrous toluene, anhydrous $\mathrm{C}_{6} \mathrm{~F}_{6}$ (1.05 equivalent), and "anhydrous" $\mathrm{TBAF}$ (1M solution in THF, 1 mole \%) were combined in a vacuum flask containing a magnetic stir bar. The vessel was sealed and the contents stirred at room temperature for 48 hours. Methanol was added to precipitate the polymer. The polymer was re-dissolved in toluene and reprecipitated with methanol, then dried under reduced pressure overnight to yield a yellow solid.

Method 2 (TBAF, Benchtop conditions).

In open air in a small vial containing a magnetic stir bar, monomer 1c, toluene, $\mathrm{C}_{6} \mathrm{~F}_{6}(1.05$ equivalent), and TBAF trihydrate $(\sim 1 \mathrm{~mol} \%)$ were combined. The vial was sealed to minimize evaporation, and the mixture was stirred at room temperature for 48 hours. Methanol was added to precipitate the polymer. The polymer was re-dissolved in toluene and re-precipitated with methanol, then dried under reduced pressure overnight to yield a yellow solid.

Method 3 (TMAF, anhydrous conditions).

In an argon-filled glove box, monomer 1, anhydrous toluene, anhydrous $\mathrm{C}_{6} \mathrm{~F}_{6}$ (1.05 equivalent), and anhydrous $\mathrm{TMAF}$ (dried at $130^{\circ} \mathrm{C}$ under vacuum for 48 hours, $5 \mathrm{~mol} \%$ ) were combined in a vacuum flask containing a magnetic stir bar. The vessel was sealed and the contents stirred at room temperature for 48 hours. Methanol was added to precipitate the polymer. The polymer was re-dissolved in toluene and re-precipitated with methanol, then dried under reduced pressure overnight to yield a yellow solid.

Method 4 (CsF/18-crown-6 anhydrous conditions).

In an argon-filled glove box, monomer 1 , anhydrous toluene, anhydrous $\mathrm{C}_{6} \mathrm{~F}_{6}$ (1.05 equivalent), and anhydrous CsF and 18 -crown-6 (vacuum dried beforehand, 10 mole $\%$ and $20 \mathrm{~mol} \%$ respectively) were combined in a vacuum flask containing a magnetic stir bar. The vessel was sealed and the contents stirred at $60^{\circ} \mathrm{C}$ for 200 hours. Methanol was added to precipitate the polymer. The polymer was redissolved in toluene and re-precipitated with methanol, then dried under reduced pressure overnight to yield a yellow solid.

Poly $1 b$ (Method 1). Monomer $1 \mathbf{b}(200 \mathrm{mg}, 0.223 \mathrm{mmol})$, toluene $(5 \mathrm{ml}), \mathrm{C}_{6} \mathrm{~F}_{6}(27 \mu \mathrm{L}, 0.234 \mathrm{mmol}, 1.05$ equivalent), and dry TBAF (1M solution in THF, $2.2 \mu \mathrm{L}, 1 \mathrm{~mole} \%)$. Yield: $176 \mathrm{mg}, 88 \% .{ }^{1} \mathrm{H}$ NMR $\left(400 \mathrm{MHz}, \mathrm{CDCl}_{3}\right) \delta 4.15(\mathrm{br}, 8 \mathrm{H}), 1.9(\mathrm{~m}, 4 \mathrm{H}), 1.15(\mathrm{~m}$, 12H), $1.24(\mathrm{~m}, 24 \mathrm{H}) 0.88(36 \mathrm{H}, \mathrm{m}) ;{ }^{13} \mathrm{C} \mathrm{NMR}\left(100 \mathrm{MHz}, \mathrm{CDCl}_{3}\right) \delta 150.01,147.79,145.31,115.47,105.12,96.40,84.03,73.34,39.27$, $37.43,37.12,34.11,29.79,27.95,24.66,22.66,22.56,22.33,19.62,14.06 . ;{ }^{19} \mathrm{~F} \mathrm{NMR}\left(376 \mathrm{MHz}, \mathrm{CDCl}_{3}\right) \delta-136.95(\mathrm{br}) ; \mathrm{M}_{\mathrm{n}}(\mathrm{GPC}$ vs PS $)=$ $83.3 \mathrm{kDa}[\mathrm{PDI}=3.76]$

Poly 1c (Method 1): Monomer 1c $(205 \mathrm{mg}, 0.2617 \mathrm{mmol})$, dry toluene $(5 \mathrm{ml})$, dry $\mathrm{C}_{6} \mathrm{~F}_{6}(32 \mu \mathrm{L}, 0.2752 \mathrm{mmol}, 1.05$ equivalent), and anhydrous TBAF (1M solution in THF, $2.6 \mu \mathrm{L}, 1 \mathrm{~mole} \%)$. Yield: $154 \mathrm{mg}, 75 \% .{ }^{1} \mathrm{H} \mathrm{NMR}\left(400 \mathrm{MHz}, \mathrm{CDCl}_{3}\right) \delta 3.93(\mathrm{~m}, 7 \mathrm{H}), 1.77(\mathrm{~m}, 4 \mathrm{H})$, $1.44(\mathrm{~m}, 32 \mathrm{H}), 0.86(\mathrm{~m}, 24 \mathrm{H}) ;{ }^{19} \mathrm{~F}$ NMR $\left(376 \mathrm{MHz}, \mathrm{CDCl}_{3}\right) \delta-136.62(\mathrm{br}) ;{ }^{13} \mathrm{C}\left(100 \mathrm{MHz}, \mathrm{CDCl}_{3}\right) \delta 150.19,147.69,145.29,114.46$, 105.07, 96.27, , 83.84, 77.93, 40.45, 30.19, 29.16, 23.46, 23.06, 13.94, 10.88.; $\mathrm{M}_{\mathrm{n}}(\mathrm{GPC}$ vs PS $)=34.7 \mathrm{kDa}$ [PDI =2.3]

Poly 1c (Method 2). Monomer 1c (120 mg,0.153 mmol), toluene (3 ml), $\mathrm{C}_{6} \mathrm{~F}_{6}(18.68 \mu \mathrm{L}, 0.153 \mathrm{mmol})$, and TBAF-trihydrate $(\sim 1 \mathrm{~mol} \%)$. Yield: $100 \mathrm{mg}, 83 \%$. ${ }^{1} \mathrm{H}$ NMR $\left(400 \mathrm{MHz}, \mathrm{CDCl}_{3}\right) \delta 3.98(\mathrm{~m}, 8 \mathrm{H}), 3.5(\mathrm{~s}, 0.17 \mathrm{H}, \mathrm{C} \equiv \mathrm{CH}$ end-groups), $1.77(\mathrm{~m}, 4 \mathrm{H}), 1.38(\mathrm{~m}, 32 \mathrm{H}), 0.86$ $(\mathrm{m}, 24 \mathrm{H}) ;{ }^{19} \mathrm{~F}$ NMR $\left(376 \mathrm{MHz}, \mathrm{CDCl}_{3}\right) \delta-136.65(\mathrm{br}) ;{ }^{13} \mathrm{C}\left(100 \mathrm{MHz}, \mathrm{CDCl}_{3}\right) \delta 150.18,147.7,145.24,114.45,105.07,96.29,83.82,76.68$, $40.38,30.18,29.15,23.45,23.05,14.04,10.97 . \mathrm{M}_{\mathrm{n}}(\mathrm{GPC}$ vs PS $)=8.9 \mathrm{kDa}[\mathrm{PDI}=2.09]$

Poly 1c (Method 3). Monomer 1c $(52.9 \mathrm{mg}, 0.0675 \mathrm{mmol})$, dry THF $(0.5 \mathrm{ml})$, dry $\mathrm{C}_{6} \mathrm{~F}_{6}(9.42 \mu \mathrm{L}, 0.081 \mathrm{mmol}, 1.2 \mathrm{equivalent})$, and dry TMAF (1 mole\%). Yield: 39mg, 73.5\%. ${ }^{1} \mathrm{H}$ NMR (400 MHz, $\left.\mathrm{CDCl}_{3}\right) \delta 3.98(\mathrm{~m}, 8 \mathrm{H}), 1.78(\mathrm{~m}, 4 \mathrm{H}), 1.48(\mathrm{~m}, 32 \mathrm{H}), 0.86(\mathrm{~m}, 24) ;{ }^{13} \mathrm{C}(100$ $\left.\mathrm{MHz}, \mathrm{CDCl}_{3}\right) \delta 150.18,147.67,145.24,114.45,105.07,96.29,83.82,76.68,40.38,30.18,29.15,23.45,23.05,14.04,10.97 .{ }^{19} \mathrm{~F} \mathrm{NMR}(376$ $\left.\mathrm{MHz}, \mathrm{CDCl}_{3}\right) \delta-136.56$ (br). $\mathrm{M}_{\mathrm{n}}(\mathrm{GPC}$ vs PS) $=153 \mathrm{kDa}[\mathrm{PDI}=1.54]$

Poly 1c (Method 4): Monomer 1c $(200 \mathrm{mg}, 0.255 \mathrm{mmol})$, dry toluene (5 ml), dry CsF (3.87 mg, $0.0255 \mathrm{mmol})$, dry 18-crown-6 (13.5 $\mathrm{mg}, 0.051 \mathrm{mmol})$, and dry $\mathrm{C}_{6} \mathrm{~F}_{6}\left(31 \mu \mathrm{L}, 0.27 \mathrm{mmol}, 1.05\right.$ equivalent). Yield: $196 \mathrm{mg}, 98 \%$. ${ }^{1} \mathrm{H} \mathrm{NMR}\left(400 \mathrm{MHz}, \mathrm{C}_{7} \mathrm{D}_{8}\right) \delta 4.13(\mathrm{~m}, 8 \mathrm{H}), 3.2$ $\left(\mathrm{s}, 0.22 \mathrm{H}, \mathrm{C} \equiv \mathrm{CH}\right.$ end-groups), $1.97(\mathrm{~m}, 4 \mathrm{H}), 1.5(\mathrm{~m}, 32 \mathrm{H}), 0.94(\mathrm{~m}, 24 \mathrm{H}), 0.1\left(\mathrm{~s}, 0.17 \mathrm{H}\right.$ TMS end groups); ${ }^{13} \mathrm{C} \mathrm{NMR}\left(100 \mathrm{MHz}, \mathrm{C}_{7} \mathrm{D}_{8}\right) \delta$ $150.19,77.93,40.38,30.19,29.15,23.05,14.023,10.97 .{ }^{19} \mathrm{~F}$ NMR $\left(376 \mathrm{MHz}, \mathrm{C}_{7} \mathrm{D}_{8}\right) \delta-136.65(\mathrm{br}) ; \mathrm{M}_{\mathrm{n}}(\mathrm{GPC}$ vs PS) $=36.3 \mathrm{kDa}[\mathrm{PDI}=$ 2.62]

NMR Scale Polymerizations:

In an argon-filled glove box, Monomer 1c $(60 \mathrm{mg}, 0.0765 \mathrm{mmol})$, toluene-d8 $(0.6 \mathrm{ml})$, dry $\mathrm{C}_{6} \mathrm{~F}_{6}(9.35 \mu \mathrm{L}, 0.0765 \mathrm{mmol}, 1.05$ equivalent $)$ were combined in an NMR tube. ${ }^{1} \mathrm{H}$ and ${ }^{19} \mathrm{~F}$ NMR spectra were measured $(\mathrm{t}=0 \mathrm{sec})$. Then anhydrous TBAF $(1 \mathrm{M}$ solution in THF, $0.76 \mu \mathrm{L}, 1$ mole \%) was added and ${ }^{1} \mathrm{H}$ and ${ }^{19} \mathrm{~F}$ NMR spectra were measured at regular intervals. After the experiment methanol was added to precipitate the polymer. The polymer was re-dissolved in toluene and re-precipitated with methanol, then dried under vacuum overnight to yield bright yellow polymer $(35 \mathrm{mg}, 58 \%) . \mathrm{M}_{\mathrm{n}}(\mathrm{GPC}$ vs $\mathrm{PS})=24.4 \mathrm{kDa}[\mathrm{PDI}=2.27]$ 


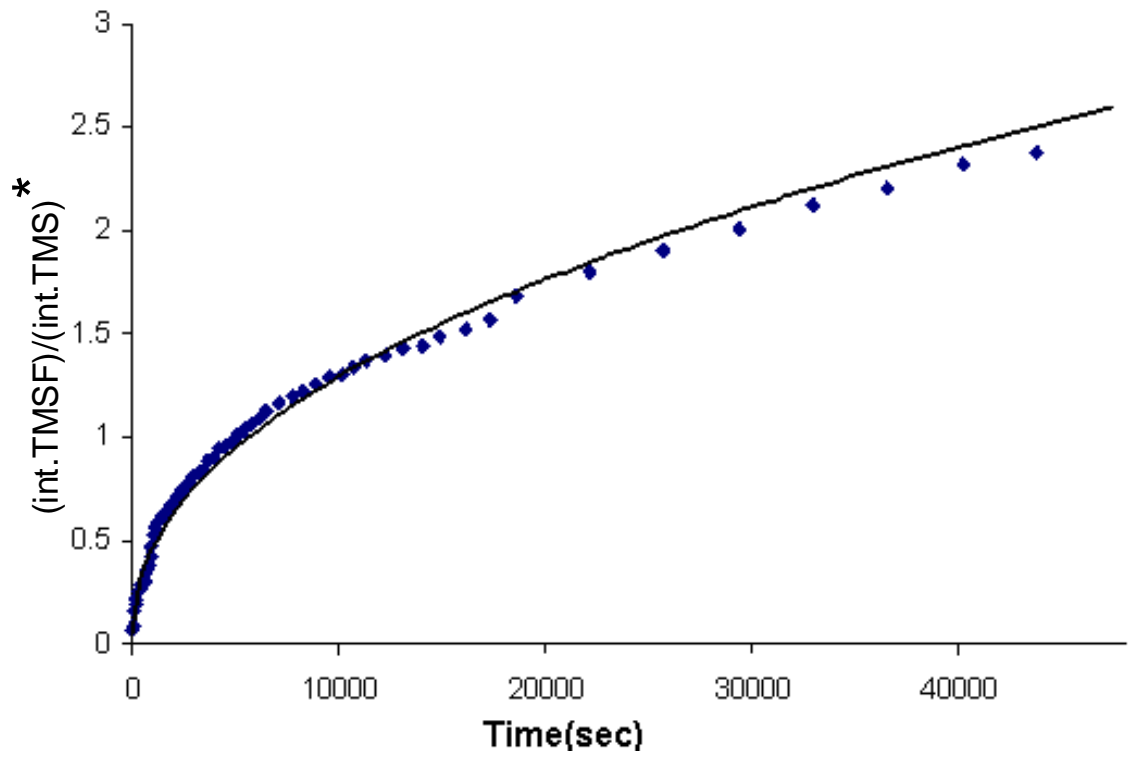

Figure SI 1: Plot of conversion over time for the copolymerization of $\mathbf{1 b}$ and $\mathrm{C}_{6} \mathrm{~F}_{6}$ in toluene- ${ }_{\mathrm{d} 8}$ catalyzed by TBAF $(1 \mathrm{~mole} \%)$. $*$ the ratio of the integrals of ${ }^{1} \mathrm{H}$ NMR signals from TMSF byproduct to unreacted TMS groups. Initial concentration of $\mathbf{1 b}=\mathrm{C}_{6} \mathrm{~F}_{6}=0.028 \mathrm{M}$, which is 4-5 times lower than any of the reported "prep scale" polymerizations.

Note: An initial monomer concentration of $0.13 \mathrm{M}$ resulted in $>70 \%$ conversion before the first spectrum acquisition ( $7 \mathrm{minutes}$ ).

\section{Stoichiometric imbalance studies:}

In an argon-filled glove box, monomer 1 , anhydrous toluene, anhydrous $\mathrm{C}_{6} \mathrm{~F}_{6}(1.0,1.2,1.4,1.6,1.8,2.0,3.0$ equivalent), and anhydrous TMAF $\left(1 \mathrm{~mol} \%\right.$, dried at $130^{\circ} \mathrm{C}$ under vacuum for 48 hours) were combined in a vial with TEFLON® cap and containing a magnetic stir bar. The vial was sealed and the contents stirred at room temperature for 48 hours. Methanol was added to precipitate the polymer. The polymer was re-dissolved in toluene and re-precipitated with methanol, then dried under reduced pressure overnight to yield the polymer. ${ }^{1} \mathrm{H}$ and ${ }^{19} \mathrm{~F}$ NMR spectral data were consistent with those recorded below. Relative number-average molecular weights $\left(\mathrm{M}_{\mathrm{n}}\right)$ were estimated by GPC (versus polystyrene standards). GPC is a measure of hydrodynamic volume, and inflated $\mathrm{M}_{n}$ values are obtained when comparing semi-rigid polymers like PPE's to coiled polystyrene standards. It has been shown that GPC with polystyrene calibration overestimates $M_{n}$ of oligo(arylene ethynylene)s by a factor of 1.5-3. ${ }^{3}$ Therefore, our measured $M_{n}$ values were converted to numberaverage degrees of polymerization $\left(\mathrm{P}_{n}\right)$ by dividing $\mathrm{M}_{n}$ by the formula weight of the repeating unit, then further dividing by a factor of two to compensate for overestimation by GPC. This treatment gives reasonably good agreement between $P_{n}$ estimated by GPC and NMR. See Figure SI 10 for an example of estimation of $\mathrm{P}_{\mathrm{n}}$ from NMR end-group analysis.

Table SI 1: Stoichiometric imbalance studies of polymer 1c. *see figure SI 10.

\begin{tabular}{|c|c|c|c|c|c|}
\hline$\left[\mathbf{C}_{6} F_{6}\right] /[1 \mathbf{c}]$ & $\begin{array}{c}\text { Predicted } \mathbf{P}_{\mathbf{n}} \\
\text { (Carothers } \\
\text { Equation) }\end{array}$ & $\begin{array}{c}\mathbf{P}_{\mathbf{n}} \text { from GPC } \\
\left(\mathbf{P}_{\mathbf{n}} / \mathbf{2}\right)\end{array}$ & $\begin{array}{c}\mathbf{P}_{\mathbf{n}} \text { from NMR } \\
\text { end group } \\
\text { analysis* }\end{array}$ & $\begin{array}{c}\text { Average of } \mathbf{P}_{\mathbf{n}} \\
\text { from } \\
\text { NMR \& GPC }\end{array}$ & $\begin{array}{c}\text { Ratio of } \\
\text { estimated to } \\
\text { predicted } \mathbf{P}_{\mathbf{n}}\end{array}$ \\
\hline 1.2 & 11 & 99 & 78 & 88.5 & 8.0 \\
\hline 1.4 & 6 & 59 & 46 & 52.5 & 8.7 \\
\hline 1.6 & 4.34 & 52 & 40 & 46 & 10.6 \\
\hline 1.8 & 3.5 & 46 & 37 & 41.5 & 11.9 \\
\hline 2.0 & 3 & 23 & 16 & 19.5 & 6.5 \\
\hline 3.0 & 2 & 7 & 10 & 8.5 & 4.3 \\
\hline
\end{tabular}


Model Reaction to ascertain reactivity of hexafluorobenzene:

In an argon-filled glove box, trimethyl(2-phenylethynyl)silane (2) $(1 \mathrm{gm}, 5.73 \mathrm{mmol}), 30 \mathrm{ml}$ anhydrous toluene, anhydrous $\mathrm{C}_{6} \mathrm{~F}_{6}(1.0$ equivalent, $667 \mu \mathrm{L})$, and TBAF (1M solution in THF, 1 mole \%) were combined in a vacuum flask containing a magnetic stir bar. The vessel was sealed and the contents stirred at room temperature for 12 hours. The solvent was removed under reduced pressure and the product mixture was separated by column chromatography (silica gel, pentane $/ \mathrm{CH}_{2} \mathrm{Cl}_{2}$ 5:1) into the following products $(\%)=$ Yields based on 2:<smiles>[R]c1c([R])c(F)c([R]([H])([H])O[Na])c([R])c1F</smiles><smiles>Fc1c(F)c(F)c(C#Cc2ccccc2)c(F)c1F</smiles>
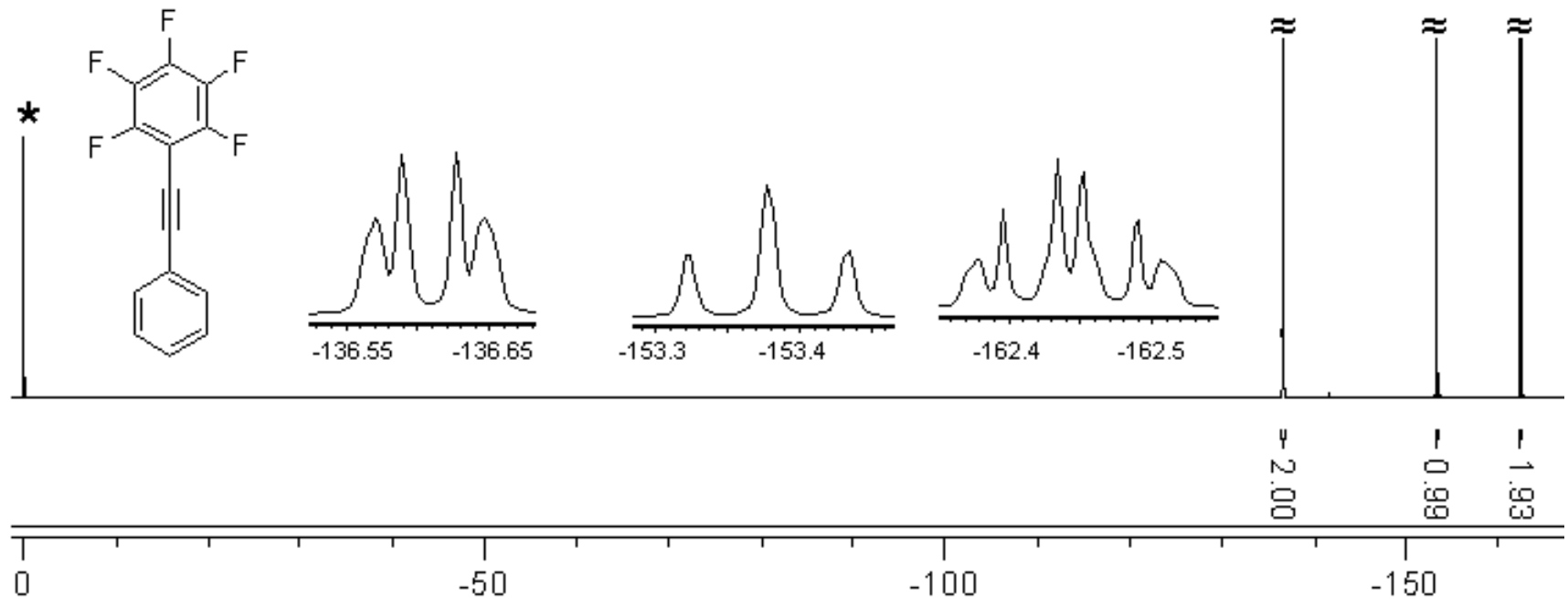

Figure SI 2: ${ }^{19} \mathrm{~F}$ NMR spectrum of compound $4\left(25^{\circ} \mathrm{C}, \mathrm{CDCl}_{3},{ }^{*} \mathrm{CCl}_{3} \mathrm{~F}\right) .{ }^{1} \mathrm{H} \mathrm{NMR}\left(400 \mathrm{MHz}, \mathrm{CDCl}_{3}\right) \delta: 7.38(\mathrm{~m}, 3 \mathrm{H}), 7.56(\mathrm{~m}, 2 \mathrm{H}){ }^{19} \mathrm{~F}$ NMR (400MHz, $\left.\mathrm{CDCl}_{3}\right) \delta$ : $-136.6(\mathrm{~m}, 2 \mathrm{~F}),-153.378(\mathrm{t}, 1 \mathrm{~F}),-162.44(\mathrm{~m}, 2 \mathrm{~F})$. HRMS calculated for $\mathrm{C}_{14} \mathrm{H}_{5} \mathrm{~F}_{5}\left(\mathrm{M}^{+}\right) 268.0305$, found 268.0303 .

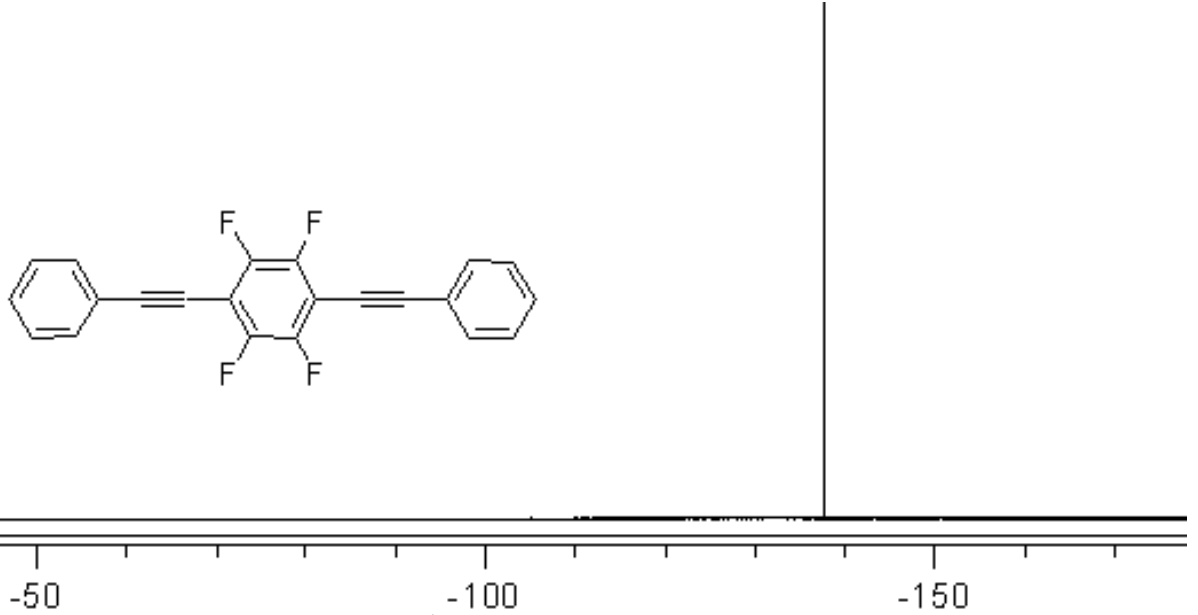

Figure SI 3: ${ }^{19} \mathrm{~F}$ NMR spectrum of compound $5\left(25^{\circ} \mathrm{C}, \mathrm{CDCl}_{3},{ }^{*} \mathrm{CFCl}_{3}\right) .{ }^{1} \mathrm{H} \mathrm{NMR}\left(400 \mathrm{MHz}, \mathrm{CDCl}_{3}\right) \delta: 7.39(\mathrm{~m}, 3 \mathrm{H}), 7.7 .59(\mathrm{~m}, 2 \mathrm{H}){ }^{19} \mathrm{~F}$ NMR (400MHz, $\left.\mathrm{CDCl}_{3}\right) \delta$ : -137.758 (s). HRMS calculated for $\mathrm{C}_{22} \mathrm{H}_{10} \mathrm{~F}_{4}\left(\mathrm{M}^{+}\right)$350.0713, found 350.0717. 


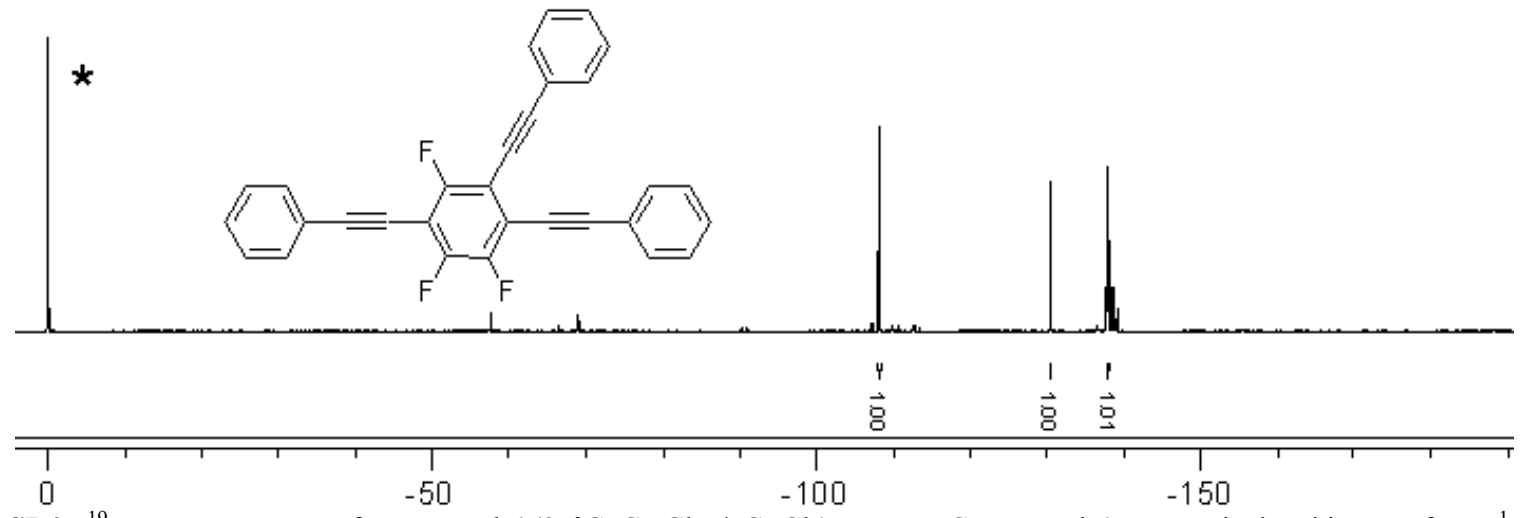

Figure SI 4: ${ }^{19} \mathrm{~F}$ NMR spectrum of compound $6\left(25^{\circ} \mathrm{C}, \mathrm{CDCl}_{3}, * \mathrm{CFCl}_{3}\right)$. Note: Compound 6 was not isolated in pure form. ${ }^{1} \mathrm{H}$ NMR $\left(400 \mathrm{MHz}, \mathrm{CDCl}_{3}\right) \delta: 7.37(\mathrm{~m}, 3 \mathrm{H}), 7.59(\mathrm{~m}, 2 \mathrm{H}){ }^{19} \mathrm{~F} \mathrm{NMR}\left(400 \mathrm{MHz}, \mathrm{CDCl}_{3}\right) \delta:-108.13(\mathrm{~d}, 1 \mathrm{~F}),-130.48(\mathrm{~d}, 1 \mathrm{~F}), 137.96(\mathrm{dd}, 1 \mathrm{~F}) . \mathrm{HRMS}$ calculated for $\mathrm{C}_{30} \mathrm{H}_{15} \mathrm{~F}_{3}\left(\mathrm{M}^{+}\right)$432.1120, found 432.1135 .
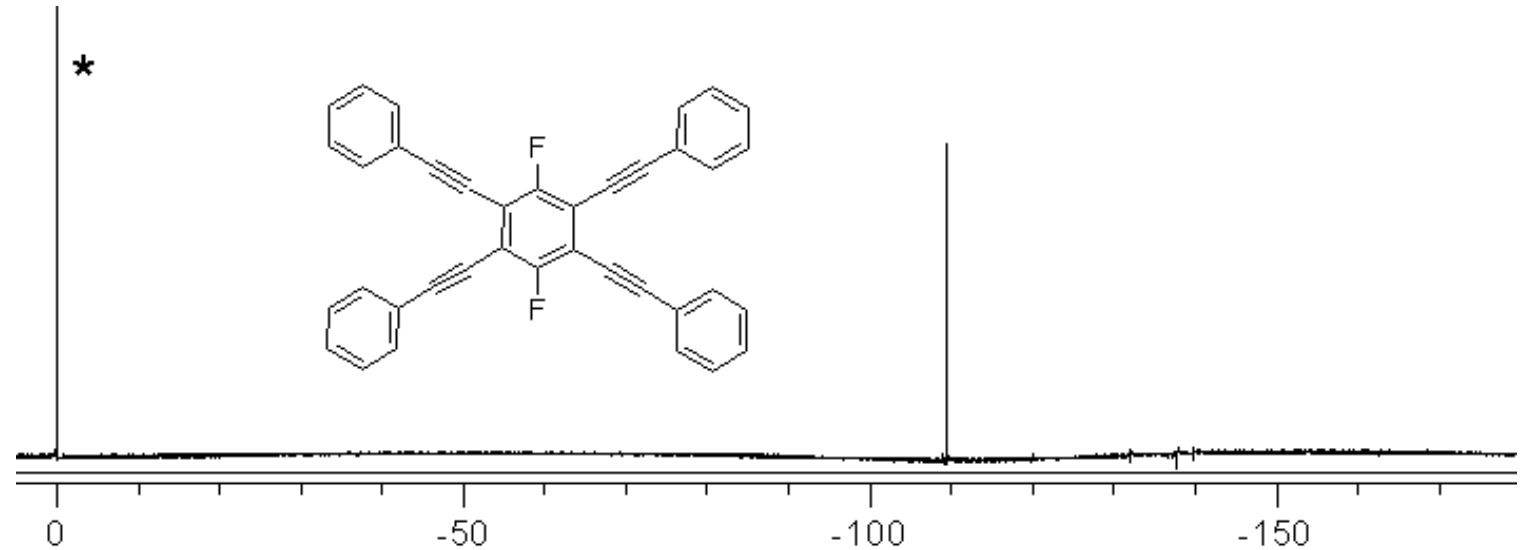

Figure SI 5: ${ }^{19} \mathrm{~F}$ NMR spectrum of compound $7\left(25^{\circ} \mathrm{C}, \mathrm{CDCl}_{3},{ }^{*} \mathrm{CFCl}_{3}\right) .{ }^{1} \mathrm{H} \mathrm{NMR}\left(400 \mathrm{MHz}, \mathrm{CDCl}_{3}\right) \delta: 7.35(\mathrm{~m}, 3 \mathrm{H}), 7.6(\mathrm{~m}, 2 \mathrm{H}){ }^{19} \mathrm{~F}$ NMR (400MHz, $\left.\mathrm{CDCl}_{3}\right) \delta$ : -109.358 (s). HRMS calculated for $\mathrm{C}_{38} \mathrm{H}_{20} \mathrm{~F}_{2}\left(\mathrm{M}^{+}\right)$514.1527, found 514.1531.

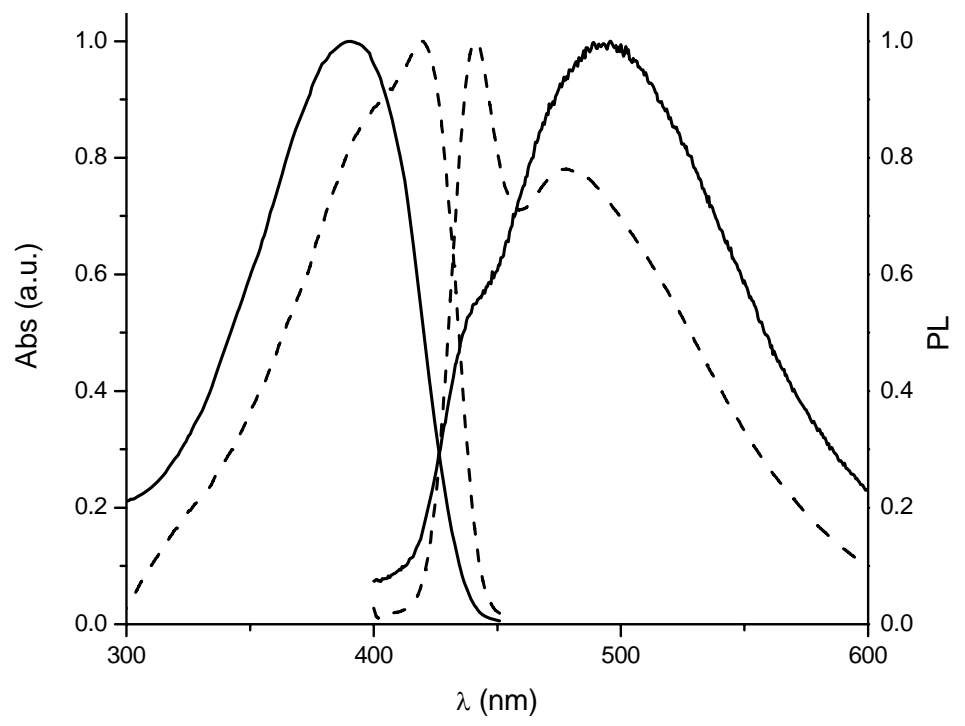

Figure SI 6: Solution absorption (Abs, $10^{-6} \mathrm{M}$ THF) and photoluminescence (PL, $10^{-8} \mathrm{M}$ THF) spectra of poly1b (dashed) and poly1c (solid). 

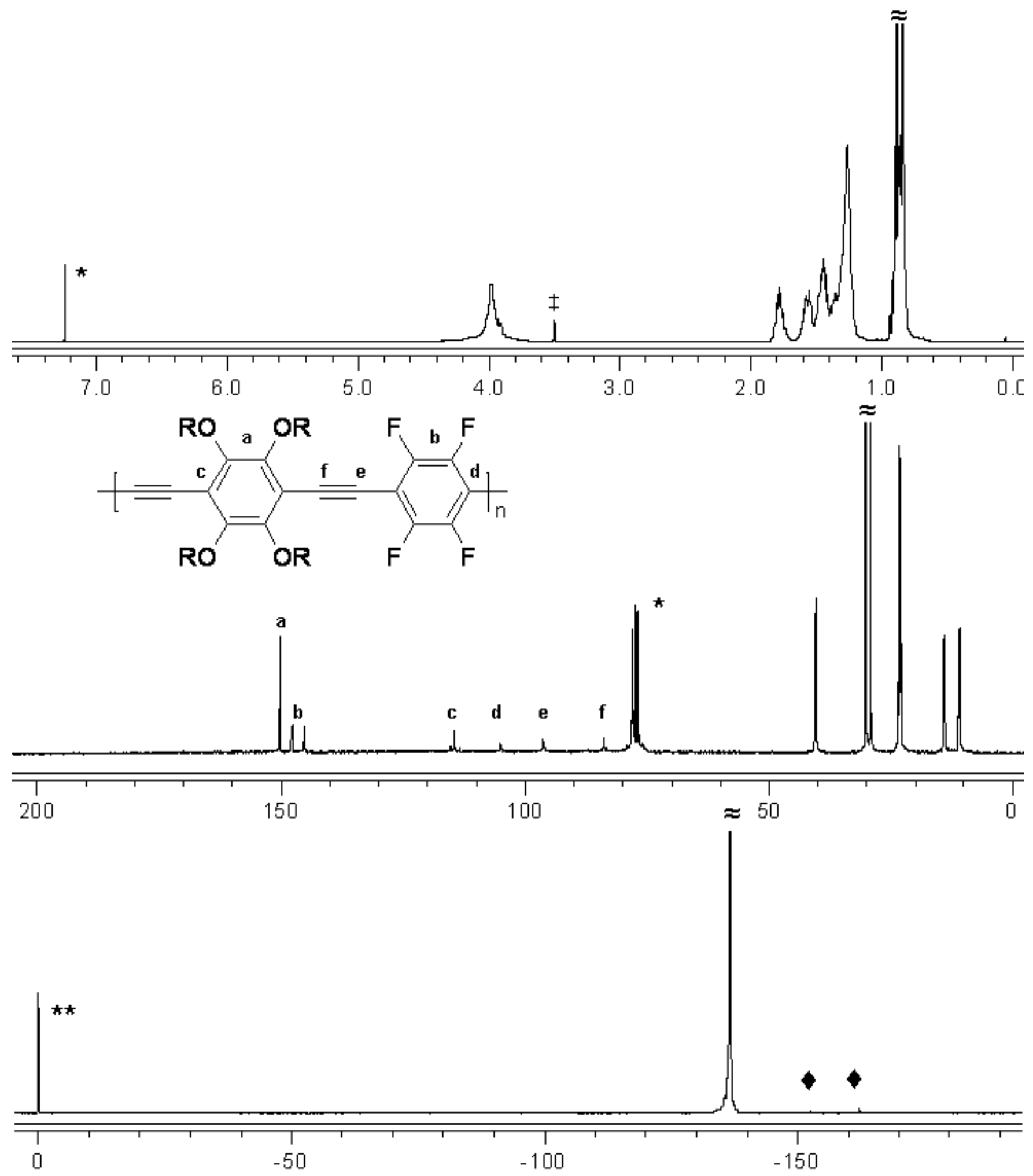

Figure SI 7: ${ }^{1} \mathrm{H},{ }^{13} \mathrm{C},{ }^{19} \mathrm{~F}$ NMR $\left(25^{\circ} \mathrm{C}, \mathrm{CDCl}_{3}\right)$ spectra of Poly 1c obtained by Method 1. ( * solvent, ${ }^{* *} \mathrm{CCl}_{3} \mathrm{~F}, \sharp \mathrm{C} \equiv \mathrm{CH}$ end group, $\mathrm{C}_{6} \mathrm{~F}_{5}$ endgroups) 

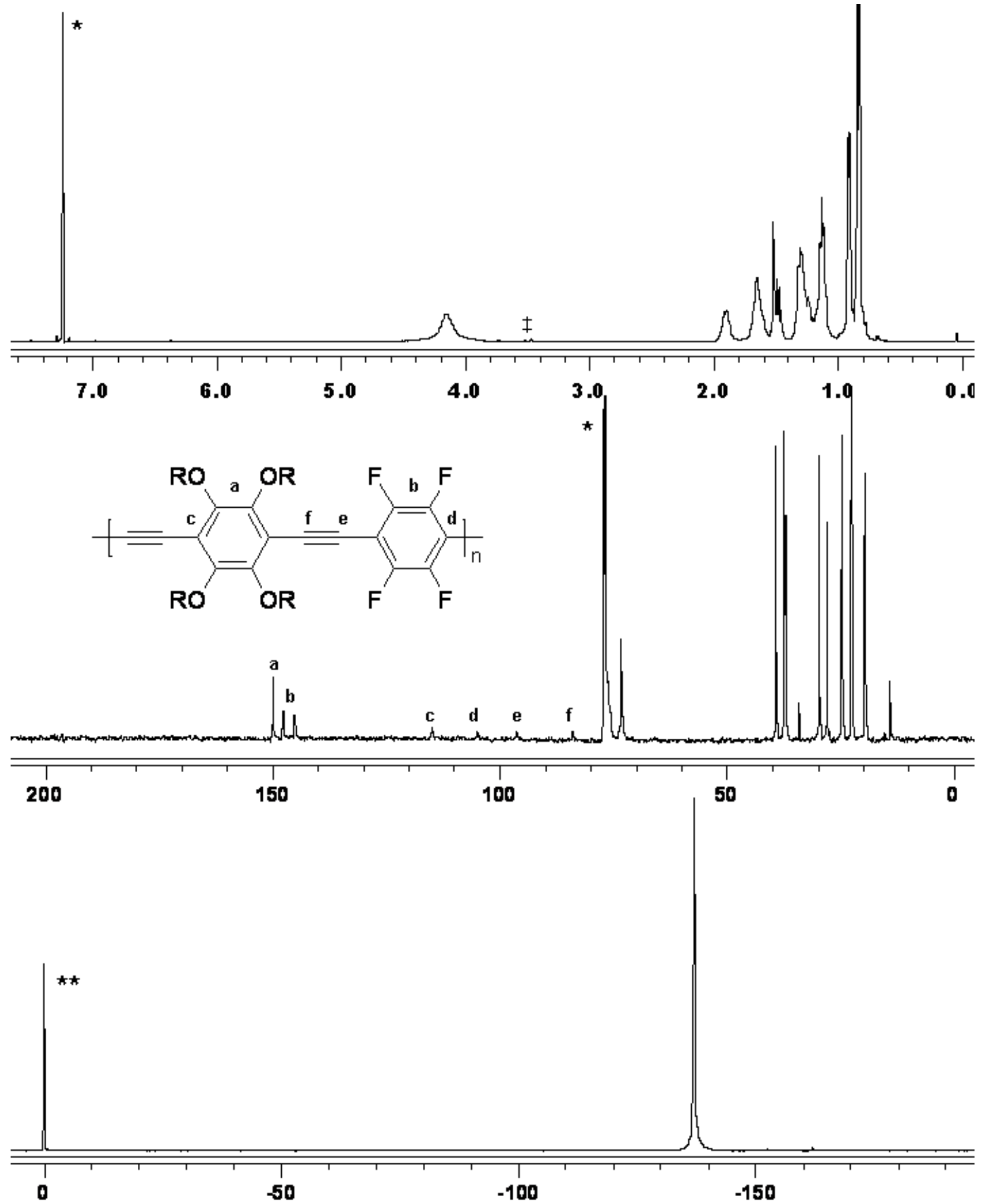

Figure SI 8: ${ }^{1} \mathrm{H},{ }^{13} \mathrm{C},{ }^{19} \mathrm{~F}$ NMR $\left(25^{\circ} \mathrm{C}, \mathrm{CDCl}_{3}\right)$ spectra of Poly $\mathbf{1 b}$ obtained by Method 1. ( * solvent, ${ }^{* *} \mathrm{CCl}_{3} \mathrm{~F}, \ddagger \mathrm{C} \equiv \mathrm{CH}$ end group) 


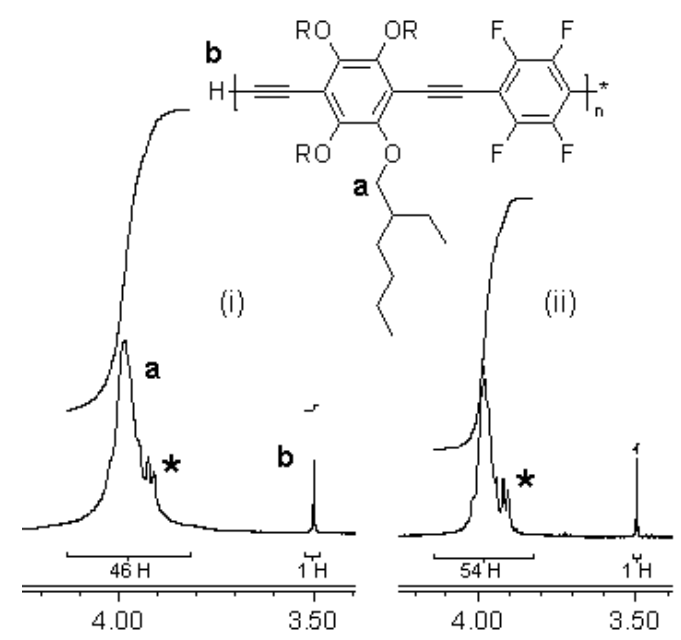

Figure SI 9: Partial ${ }^{1} \mathrm{H}$ NMR spectra $\left(25^{\circ} \mathrm{C}, \mathrm{CDCl}_{3}\right)$ of Poly 1c showing relative integrals of signals arising from $\mathrm{C} \equiv \mathrm{CH}$ endgroups and $\mathrm{OCH}_{2}$ protons of side chains. The conditions were (i) Method 2: TBAF, off-the-shelf toluene, (ii) Method 1: TBAF, anhydrous toluene, (iii) Method 3: TMAF, anhydrous toluene. The degree of protiodesilylation, which limits molecular weight, is greatly reduced with anhydrous TMAF relative to TBAF as catalyst. The commercial TBAF solution ( $1 \mathrm{M} \mathrm{THF})$ is $5 \%$ water (v/v) and likely contains acidic products of Hoffman elimination. ${ }^{*} \mathrm{OC} \underline{H}_{2}$ protons of end-groups.

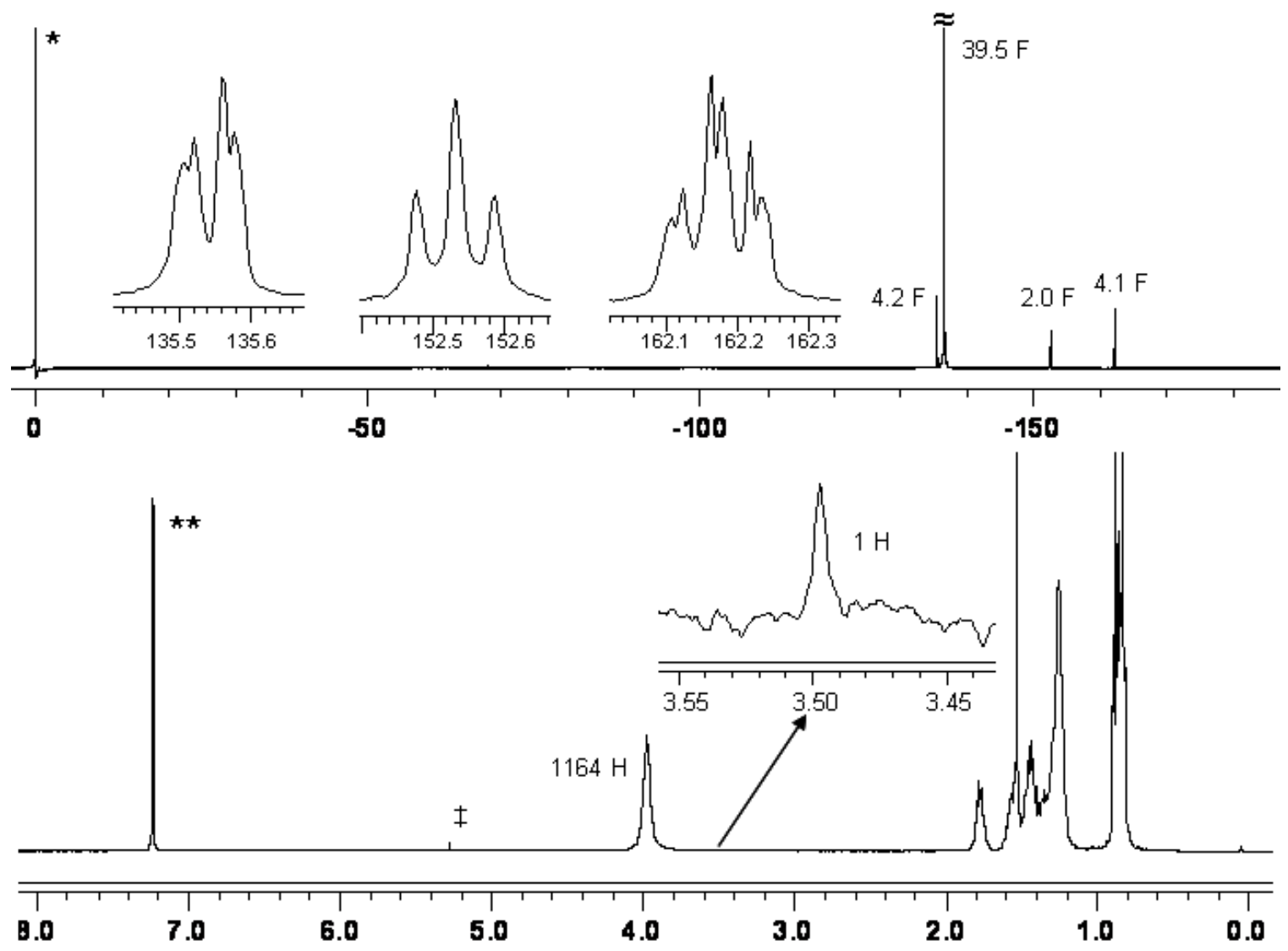

Figure SI 10: ${ }^{19} \mathrm{~F}$ and ${ }^{1} \mathrm{H}$ NMR spectra $\left(25^{\circ} \mathrm{C}, \mathrm{CDCl}_{3}\right)$ of Poly1c (Method 3, stoichiometric study with $\left[\mathrm{C}_{6} \mathrm{~F}_{6}\right] /[\mathbf{1 c}]=3, * \mathrm{CCl}_{3} \mathrm{~F}, * *$ solvent, $\$ \mathrm{CH}_{2} \mathrm{Cl}_{2}$ ). Compare chemical shifts and multiplicities of $\mathrm{C}_{6} \mathrm{~F}_{4}$ (repeating unit) and $\mathrm{C}_{6} \mathrm{~F}_{5}$ (end groups) of the ${ }^{19} \mathrm{~F}$ NMR spectra to those of compounds 5 and $\mathbf{4}$ respectively. Sample calculation for determining $\mathbf{P}_{\mathbf{n}}$ by $\mathbf{N M R}$ end group analysis: There is one $\mathrm{C} \equiv \mathrm{CH}$ endgroup (3.5 ppm) for every $1164 \mathrm{H} / 8 \mathrm{H}=146$ repeat units. There are two $\mathrm{C}_{6} \mathrm{~F}_{5}$ end groups for every $39.5 / 4=9.9 \mathrm{C}_{6} \mathrm{~F}_{4}$ repeat units. Normalization of the total number of endgroups per repeat units yields 2.03 total endgroups per 9.9 repeat units: $\mathrm{P}_{\mathrm{n}} \sim 10$.

\section{References:}

1. Woody, K.B.; Watson, M.D., Bullock, J.E.; Parkin, S.R. Macromolecules, 2007, 40,4470-73

2. Keegstra, Erik. M.D; Huisman, B.H.; Paardekooper, E.M.; Hoogesteger, F.J.; Zwikker, J.W.; Jenneskens, L. W.; Kooijman, H.;

Schouten, A.; Veldman, N.; Spek,A.L. J.Chem.Soc,Parkin Trans.2, 1996,2, 229-40

3. Tour, J.M. Chem. Rev. 1996, 96, 537-553. 